\title{
From Eating to Emotional Eating
}

\author{
Pnina Hertz \\ Hadassah Medical Center, Jerusalem, Israel
}

\begin{abstract}
Feeding and eating disorders are common in children, and may begin in infancy, in infants who nurse or are bottle-fed. The challenges and difficulties involved in feeding and eating are diverse and characterized differently at each developmental stage. There may be a continuum between the lack of parental sensitivity in feeding infants and children and eating disorders in adolescence. In other cases, eating disorders, such as emotional eating in adolescence, are a response to environmental-familial complexity and may have genetic components without a primary source in the early stages of development. In the case described in this article, Dalia was treated for about a year for emotional eating, which resulted in excess weight. Other aspects of emotional, social, and interpersonal issues were discussed. Treatment of the symptom was not achieved but due to the therapeutic flexibility and willingness to switch between therapeutic approaches, other important goals were achieved. Treatment using positive psychology with emphasis on empowerment may be beneficial to adolescents while personality is shaped as compared with cognitive-behavioral therapy that requires a long-term commitment.
\end{abstract}

Keywords: feeding disorders, eating disorders, emotional eating, cognitive behavioral therapy, positive psychology

\section{Introduction}

Eating is a simple and natural action. It starts at birth with nursing. Eating improves with time and enables one to eat food with different textures, tastes, and temperatures.

Normal eating means putting food into one's mouth and its digestion. The primary goal of putting food into one's body is physical maintenance for survival and quality of life. This goal is fundamental but come with other diverse goals. Eating and being exposed to a variety of foods is pleasurable, stimulates the senses, strengthens parts of the mouth, and develops mouth functions related to eating.

Eating also has a social component and eating together makes it possible to share the enjoyment of food. There is also a ritual aspect to family meals on holidays and special occasions. Different societies have different perceptions, prohibitions, and limitations on specific foods, connected to customs and traditions-within religious or social contexts.

In contemporary western society today, the ideal body type is slim and the pressure to maintain it primarily influences girls and women. Young girls can also be preoccupied by this beauty ideal. The age of preoccupation with body image and thinness gets younger, a matter of concern for both the family and the community.

\section{Eating and Emotion in the Beginning of Life}

Seemingly, eating is not connected to the world of emotions. As mentioned, people eat to survive. If we were not in daily contact with people and we lived alone, our involvement with food and eating might be

Pnina Hertz, Ph.D., Pediatric Psychologist, Hadassah Medical Center, Jerusalem, Israel. 
minimal and functional. The actuality is more complex — food and eating satisfy not only physical but also emotional needs.

Food and its consumption are meant to connect to a range of positive feelings. The simpler the involvement with food and its consumption, the more likely the emotional experience will be good, including the pleasant physical sensation of satiety and the emotions of tranquility and happiness.

Attentive feeding is based on sensitivity to the needs of the infant. The parent should feed according to the infant's needs and developmental stage and not according to the parent's wishes, outlooks desires. When the parent-infant relationship is problematic, positive experiences may be more limited, resulting in negative feelings and sensations. An infant who senses that she and her needs are not being met and is fed in a technical manner every few hours, may be frustrated, angry and even feel helpless (Anderson \& Whitaker, 2011). In this way, a disturbance of the simple feeding mechanism, which is supposed to be rewarding for feeder and infant, can develop. A food disturbance can be feeding that is too fast or too slow, feeding food unpleasant to the baby's palate, food that is not at the right temperature. In extreme cases, we are dealing with pathological parents, with at-risk characteristics of preventing basic needs, starving the baby or inventing illnesses (American Psychiatric Association, 2013). In other cases, immature parents have difficulties separating their needs and the baby's needs (Fraiberg, Adelson, \& Shapiro, 1975).

\section{Eating, Emotion, and the Cycle of Life}

The emotional experience of the infant who is fed and not well-satisfied is different than that of children, adolescents or adults. Preschoolers develop the ability and desire to choose, to resemble or be different from other children. They develop taste and texture preferences and require primary caregivers who respect their choices. They enjoy social eating but at times withdraw or are afraid of social eating. They want to and can eat independently and their autonomy in eating needs to be respected.

Involvement with body image, self-image and social image increase with age and belonging to a group. Adolescents are involved with how they look, in comparison with their peers. This relative "looking" is more apparent in girls than in boys and can result in food disturbances, some of which are serious and dangerous.

The feeling of jealousy arises with comparisons to others, accompanied by a tendency to self-criticism and self-judgment. In addition to jealousy, there may be anger and frustration due to the gap between the adolescents' aspirations, actual situation and self-perceptions in this area.

Sometimes, experiences characterized by conflict cause overeating, emotional eating, weight gain, and obesity. The familiar scenario is a circle of shame, guilt, and even despair. These feelings can increase emotional eating and consequently, negative feelings that increase in intensity and frequency. In this absurd situation, the food which comforts momentarily increases the intensity of the negative feelings which then results in eating, a seeming compensation, and the cycle repeats over and over.

The proverb, "Stolen waters are sweet" can be seen in a person dealing with prohibitions or restrictions in the area of food, primarily when facing social expectations. The environment's dictates are sometimes internalized by those trying to adapt to the ideal slimness. The more stolen and forbidden the "water", the more desired and necessary for the person struggling with the challenges of limiting and reducing food intake. The desire for food is always in the background, also when the person seemingly renounces food and is accompanied by feelings connected with the world of prohibitions. 


\section{Eating and Feeding Disturbances Among Children}

In this area, there have been many conceptual changes in changed and renewed diagnoses in different guides to psychiatric diagnoses (Zero to Three, 2016).

Use of the term "feeding disturbances" emphasizes the aspect of the feeding personality in contrast with the passive-receptive position of the infant being fed. The child's development of motor abilities and independence makes active eating possible and therefore the term "eating disturbances" can be used. Feeding disturbances are varied and include a wide category of overfeeding and a wide category of underfeeding. This distinction is especially relevant in the first years of life when food regulation is of utmost importance. At this stage the ultimate goal is daily balance and regulation in eating according to the needs that arise.

In modern life, there is a distinct phenomenon of choosing to consume specific food groups and avoid other food groups. This restriction can result in inadequate intake of food that is necessary for normal body development. Satisfying basic needs is important for long term health. Not surprisingly, therefore, the contemporary involvement with a specific diagnosis, Avoidant/Restrictive Food Intake Disorder (ARFID), that relates to children's significant restriction and abstention from certain foods. The refusal to eat a variety of foods can stem from a variety of causes, among them physical causes, oral sensory sensitivity or emotional causes - anxiety-provoking memories and conditioning connected with feeding (Chattor, 2009; Zero to Three, 2016)

The place of the main caregiver who feeds is most important. He/she is the balancing regulating agent who educates to listening and positive encounters with food, in a facilitating, accepting environment. This is a challenging role and requires the responsible choice of a balanced feeding style without overusing emotions or prizes, without limiting food or overfeeding. In a world of abundant food, where obesity is an epidemic occurring at a younger age, this role is of utmost importance (Wardle, Sanderson, Guthrie, Lorna Rapaport, \& Plomin, 2002; Anderson \& Whitaker, 2011).

\section{Emotional Eating Among Adolescents}

An adolescent can take partial or full responsibility for his meals. He is experienced as to his food intake and the environment tends to judge him on consuming unusual amounts. The term "emotional eating" focuses on how the food is consumed and the accompanying responsibility. Very possibly, the environment will harshly judge eating that stems from emptiness, boredom, sadness, or frustration.

Two additional significant factors may influence overweight and obesity: environment-food consumption habits, food storage, and food use at home - and genetics. Often, a fat person is not the only fat person in the family. In one family, there may be two fat children and two thin children and often, there will not be an apparent explanation of the difference. One cannot connect the phenomenon of excessive weight gain/obesity with the place of the child in the family, sex, and age of onset.

Even though the term "emotional eating" can be interpreted as negative and judgmental, I will use this term to be precise and explain that we are discussing eating beyond satiety, which sometimes occurs when people do not experience hunger that directs them towards appropriate eating times and consuming satisfying comfortable amounts of food. When discussing treatment processes, I will emphasize environmental support and involvement to counterbalance the weak points and complex relationship with food which some adolescents develop (Conion et al., 2019). 


\section{Case Description}

Dalia was referred to the clinic by her father to treat emotional eating and weight gain. After her parents separated, Dalia lived with her father, which was a complicated process. In the beginning of treatment, Dalia, aged thirteen, looked unconfident and uncomfortable in her body. She had gained weight and was on the border of being overweight. She was diffident and awkward and creating a significant and even strong therapeutic connection with her took time. Her shyness, sensitivity and pleasant manner were characteristic traits throughout the year-long therapy. Technical constraints determined that the therapeutic framework be bi-monthly sessions.

Throughout treatment, the connection with Dalia's father was ongoing while the connection with her mother was minimal and not initiated by her. It was clear that Dalia grew up in a family unit with many challenges. The people around her had evident strengths and individual resources. There was a significant support system and a readiness to join some of Dalia's sessions with me, following my recommendation.

Parallel to receiving psychological treatment and with the father's understanding that Dalia's overeating also had an emotional background, ongoing nutrition counseling was given, with medical supervision when necessary. Her medical tests were normal without nutritional deficits or abnormal blood results. These findings calmed and pleased those involved but possibly weakened Dalia's motivation to lose weight (because of her healthy medical status).

In the beginning of treatment, Dalia expressed the desire to lose weight and was more involved than her friends and sisters with her body and weight. We had to clarify her capabilities for the long Sisyphean process that would lead to weight loss.

The treatment approach included an intake, systematically collecting information, monthly weighing with a discussion of results and identifying the emotional range, especially identifying those emotions that lead to overeating. Awareness of the range of emotions was a new process for Dalia who was unsure of the presence and intensity of certain emotions in her daily life. Some emotions were even seen as negative and forbidden, in her perspective and in the education she had received. She learned to respect these emotions, contain them and see them as part of her generally positive beloved personality. She learned to distinguish between her desire for achievements in a certain area and her jealousy, which did not further her personally but instead focused on the other person and his achievements.

Environmental and personal data led to cognitive behavioral therapy inspired treatment and the possible use of a diary to record emotions. This was accompanied by counseling Dalia's parents on food consumption habits, eating patterns, and household mealtime practices. For a certain period, there were daily emails for communication, including a daily eating schedule. Dalia had a hard time committing to the plan and the email communication became infrequent and was used for irregular communication with Dalia or her father.

Short-term and medium-long term goals were determined. Immediate goals included empowerment, advancing in the process of self-acceptance and lessening the tendency to self-negation. Medium-term and long-term goals included developing an identity perspective that approached weight and physical state as parts of a whole personality.

The main therapeutic achievement was building a therapeutic connection based on trust, self-acceptance and enhanced self-confidence. These were expressed in Dalia's talking louder, larger handwriting, and the willingness "to take up space". 
Dalia's social abilities improved in the course of treatment. She learned to accept help and cope with learning difficulties maturely and flexibly. Her social and educational progress was done with full cooperation between the clinic staff, the family, and the educational staff and an ongoing connection with the treating nutritionist.

The year of Dalia's therapy illustrated the need to integrate the different needs of an adolescent girl in a multidisciplinary approach to treat eating patterns and their results because of the influence of emotional eating on a variety of areas and situations. The case of Dalia teaches that the treatment of adolescents in the formative stage of their personality requires flexibility, moving between therapeutic techniques and even changing therapeutic goals, which are usually determined in advance.

Dalia failed to lose weight but learned that improved coping with emotions, personal development and personality changes do not necessarily lead to physical changes.

The percentages of successful treatment of emotional eating are not high long term but should increase as the personality consolidates, and by simultaneously applying principles from positive psychology such as positive thinking, empowerment, growth and emotional steadfastness in the face of expected and unexpected challenges (Yildirim \& Belen, 2019).

\section{Discussion}

Emotional eating in adolescence is a common outcome of feeding and eating patterns at a young age. A wide range of factors contribute to this phenomenon. Genetic factors are connected to weight gain/obesity as well as the social influence of body image and its place among adolescents.

Sometimes, children and adolescents cope with emotional eating as individuals and are the exception in their families; there can however also be a genetic tendency.

Adolescence is a stage characterized by personality development. The adolescent has both his own wishes and preferences and those in comparison with his environment, primarily his peer group. The adolescent may well succeed in setting his own goals and in acting according to those set with his participation. On the other hand, persevering for long term goals is difficult, even when agreed-upon with willingness to act in achieving them. Long-term commitment in the stage of multidisciplinary changes and the creation of the personality structure is not simple.

When experiencing failure, the feelings of self-blame, shame, and even despair may appear or be overwhelming. Frustration due to frequent failures for those repeatedly dieting may result in a cumulative weight gain. This creates a complex tight circle of the individual's not listening to himself and his needs; unregulated eating becomes entrenched, increasing negative feelings. Emotional eating with possible weight gain does not have a single source; it is the outcome of physical, emotional, and social interactions.

The year of therapy with Dalia illustrates the therapeutic complexity in the development stage characterized by multi-level changes. The psychological therapies may then also be adjusted, necessitating flexibility and a variety of therapeutic techniques.

Dalia came to therapy wanting to lose weight. Her achievements were in other areas-emotional, interpersonal, social, and educational. The therapeutic experience with Dalia can be articulated: "He went looking for donkeys and found a kingdom" (Samuel 1, Chapter 9) from Bible. 


\section{References}

American Psychiatric Association. (2013). Diagnostic and Statistical Manual of Mental Disorders (5th ed.). Arlington, VA: American Psychiatric Association.

Anderson, S. E., \& Whitaker, R. C. (2011). Attachment Security and Obesity in US Preschool-Aged Children. Arch PediatrAdolesc Med, 165(3), 235-242.

Chattor, I. (2009). Sensory Food Aversions in Infants and Toddlers. Zero to Three, 44-49.

Conion, B. A., Mcginn, A. P., Isasi, C. R., Mossavar-Rahmani Y., Lounsbury, D. W., Ginsberg, M. S., ... Wylie-Rosett, J. (2019). Home, Environment Factors and Health Behaviors of Low-income, Overweight and Obese Youth. Am. J Health Behav, 43(2), 420-436.

Fraiberg, S., Adelson, E., \& Shapiro, V. (1975). Ghosts in the nursery: a psychoanalytic approach to the problems of impaired infant-mother relationships. Journal of The American Academy of Child and Adolescent Psychiatry, 14(3), 387-421.

Wardle, J., Sanderson, S., Guthrie, C. A., Lorna Rapaport, L., \& Plomin, R. (2002). Parental Feeding Style and the Inter-generational Transmission of Obesity Risk. Obesity Research, 10, 453-462.

Yildirim, M., \& Belen, H. (2019). The Role of Resilience in the Relationships between Externality of Happiness and Subjective Well-being and Flourishing: A Structural Equation Model Approach. Journal of Positive Psychology and Wellbeing, 3(1), $62-76$.

Zero to Three. (2016). DC: 0-5: Diagnostic classification of mental health and developmental health and developmental disorders of infancy and early childhood. Washington, DC: Author. 